ARTICLE

Received 7 Mar 2016 | Accepted 22 Aug 2016 | Published 6 Oct 2016

DOI: $10.1038 /$ ncomms12968

OPEN

\title{
Competitive solvent-molecule interactions govern primary processes of diphenylcarbene in solvent mixtures
}

\author{
Johannes Knorr ${ }^{1}$, Pandian Sokkar ${ }^{2}$, Sebastian Schott ${ }^{3}$, Paolo Costa ${ }^{4}$, Walter Thiel ${ }^{2}$, Wolfram Sander ${ }^{4}$, \\ Elsa Sanchez-Garcia ${ }^{2} \&$ Patrick Nuernberger ${ }^{1}$
}

Photochemical reactions in solution often proceed via competing reaction pathways comprising intermediates that capture a solvent molecule. A disclosure of the underlying reaction mechanisms is challenging due to the rapid nature of these processes and the intricate identification of how many solvent molecules are involved. Here combining broadband femtosecond transient absorption and quantum mechanics/molecular mechanics simulations, we show for one of the most reactive species, diphenylcarbene, that the decision-maker is not the nearest solvent molecule but its neighbour. The hydrogen bonding dynamics determine which reaction channels are accessible in binary solvent mixtures at room temperature. In-depth analysis of the amount of nascent intermediates corroborates the importance of a hydrogen-bonded complex with a protic solvent molecule, in striking analogy to complexes found at cryogenic temperatures. Our results show that adjacent solvent molecules take the role of key abettors rather than bystanders for the fate of the reactive intermediate.

\footnotetext{
${ }^{1}$ Physikalische Chemie II, Ruhr-Universität Bochum, 44780 Bochum, Germany. ${ }^{2}$ Max-Planck-Institut für Kohlenforschung, Kaiser-Wilhelm-Platz 1, 45470 Mülheim an der Ruhr, Germany. ${ }^{3}$ Institut für Physikalische und Theoretische Chemie, Universität Würzburg, Am Hubland, 97074 Würzburg, Germany.

${ }^{4}$ Organische Chemie II, Ruhr-Universität Bochum, 44780 Bochum, Germany. Correspondence and requests for materials should be addressed to

E.S.-G. (email: esanchez@kofo.mpg.de) or to P.N. (email: patrick.nuernberger@rub.de).
} 
C arbene chemistry is a multifaceted research area by virtue of the highly spin-dependent reactivity of carbenes ${ }^{1}$. Various spectroscopic investigations ranging from laser flash-photolysis ${ }^{2-12}$, matrix-isolation studies ${ }^{13}$ to ultrafast measurements in different environments ${ }^{14-22}$ have unveiled the role of carbenes as intermediates in (photo)chemical reactions. In particular, the reactivity of diphenylcarbene (diphenylmethylene, $\mathrm{Ph}_{2} \mathrm{C}$ ) intrigues researchers owing to the simplicity of the system accompanied by the variety of possible reaction pathways depending on whether the spin-configuration is either a singlet or a triplet state $23-25$. Singlet carbenes are assumed to insert into $\mathrm{O}-\mathrm{H}$ bonds, whereas triplet carbenes insert into $\mathrm{C}-\mathrm{H}$ bonds ${ }^{5}$. Studies by Eisenthal and coworkers ${ }^{2-11}$ have pioneered many aspects of $\mathrm{Ph}_{2} \mathrm{C}$ reactivity in solution, for example, effects of solvent polarity and selected cosolvents, but without the direct spectroscopic observation of the singlet ${ }^{1} \mathrm{Ph}_{2} \mathrm{C}$. This was possible in ultrafast studies, performed initially by the Chergui group ${ }^{14}$ and further extended by Kohler and coworkers ${ }^{15}$ in several pure solvents. The latter revealed that ultraviolet excitation of the diazo-compound precursor diphenyldiazomethane $\left(\mathrm{Ph}_{2} \mathrm{CN}_{2}\right)$ leads to formation of ${ }^{1} \mathrm{Ph}_{2} \mathrm{C}$ on a subpicosecond time scale, which can further react via intersystem crossing (ISC) to the triplet ${ }^{3} \mathrm{Ph}_{2} \mathrm{C}$ in a few hundreds of picoseconds in aprotic solvents. However, if ${ }^{1} \mathrm{Ph}_{2} \mathrm{C}$ is allowed to react with alcohols, an ultrafast intermolecular proton-transfer can occur, leading to an intermediate benzhydryl cation $\left(\mathrm{Ph}_{2} \mathrm{CH}^{+}\right)$, and eventually to a diphenylalkyl ether, the formal product of $\mathrm{O}-\mathrm{H}$ insertion. Kirmse and Steenken ${ }^{12,26}$ inferred that two alcohol molecules are needed for the protonation. Others postulated a concerted reaction pathway, possibly involving an intermediate ylide ${ }^{27-29}$. Recent findings in matrix-isolation experiments at low temperatures provided additional aspects of diphenylcarbene reactivity: in argon matrices doped with $0.5-1 \%$ methanol $(\mathrm{MeOH}),{ }^{1} \mathrm{Ph}_{2} \mathrm{C}$ (in contrast to ${ }^{3} \mathrm{Ph}_{2} \mathrm{C}$ ) forms a hydrogen-bonded complex with a $\mathrm{MeOH}$ molecule, hence refuting the assumption of a general triplet ground state ${ }^{30}$. Whereas similar results have been found when replacing the $\mathrm{MeOH}$ dopants by water molecules, an amorphous water environment enabled the isolation of stable $\mathrm{Ph}_{2} \mathrm{CH}^{+}$without the need for superacidic conditions $^{31}$.

While the behaviour of $\mathrm{Ph}_{2} \mathrm{C}$ in pure solvents has been investigated, the emphasis of our study is also on the dynamics in solvent mixtures, because the possible reaction channels of ${ }^{1} \mathrm{Ph}_{2} \mathrm{C}$ accessible under different conditions will compete with each other when the solvent environment is systematically varied. Here we combine hybrid quantum mechanics/molecular mechanics (QM/MM) calculations and advanced femtosecond pump-probe spectroscopy to unravel the fate of ${ }^{1} \mathrm{Ph}_{2} \mathrm{C}$ in binary mixtures of the protic solvent $\mathrm{MeOH}$ and the aprotic acetonitrile $(\mathrm{MeCN})$ at room temperature. By going from neat $\mathrm{MeOH}$ to pure $\mathrm{MeCN}$, a tailored solvent environment with variable distances between the carbene and the alcohol molecules is created, also aimed at 'mimicking' the $\mathrm{MeOH}$ doping of the matrix-isolation studies. The joint theoretical and experimental investigation directly visualizes the interplay and competition of the solvent molecules in the mixtures, discloses the amount of solvent molecules necessary for certain reaction pathways and allows the identification of a concerted reaction pathway that involves an intermediate complex ${ }^{1} \mathrm{Ph}_{2} \mathrm{C} \cdots$ HOMe. Our results emphasize that ultrafast photodynamics in solvent mixtures are more than just a linear combination of the behaviour in neat solvents, and that the amount of molecules of a certain solvent in the vicinity of the solute may be of critical importance for a desired reaction outcome.

\section{Results}

Computational studies. The following experimental and theoretical studies aim at understanding the competition between reaction channels accessible not only in pure solvents, but also in solvent mixtures on an ultrafast time scale. For a mechanistic understanding at the molecular level, hybrid QM/MM calculations were employed to capture the solvent effects and the specific interactions governing the stability of $\mathrm{Ph}_{2} \mathrm{C}$ (see Methods and Supplementary Table 1). We performed solvent-only simulations to validate the behaviour of the physical properties of pure $\mathrm{MeOH}$, pure $\mathrm{MeCN}$ and a mixture of $\mathrm{MeCN}: \mathrm{MeOH}$ $(80: 20 \% \mathrm{v} / \mathrm{v})$ during the simulations (Supplementary Table 1 and Supplementary Note 1). The simulations confirm that the solvent structure involves hydrogen-bonds in pure $\mathrm{MeOH}$ and that there is no well-structured solvent arrangement in pure MeCN (Supplementary Fig. 1a and Supplementary Fig. 1b). For the mixture, the simulations indicate the absence of phase separation or aggregation, which is in accordance with experimental observations (see Supplementary Fig. 1c, Supplementary Fig. 2 and Supplementary Note 2) of rather weak preferential solvation $^{32}$.

In order to elucidate whether a solvent molecule can significantly influence the balance of the $\mathrm{Ph}_{2} \mathrm{C}$ spin states, we analysed the average QM energies of ${ }^{1} \mathrm{Ph}_{2} \mathrm{C}$ and ${ }^{3} \mathrm{Ph}_{2} \mathrm{C}$ in explicit solvent (QM/MM calculations, see Supplementary Figs 3 and 4 and Supplementary Note 3). Although pure $\mathrm{MeCN}$ stabilizes the singlet more than the triplet, the latter is energetically still favoured, as in the gas phase. The situation already changes by addition of small amounts of $\mathrm{MeOH}$, reversing the singlet-triplet energy gap (see Fig. 1 and Supplementary Tables 2-4). The optimized structures reveal that $\mathrm{MeOH}$ forms a strong $\mathrm{H}$-bond with ${ }^{1} \mathrm{Ph}_{2} \mathrm{C}$, thereby lowering its energy, whereas in the case of the less polar ${ }^{3} \mathrm{Ph}_{2} \mathrm{C}$ the stabilization is much less pronounced. This behaviour is also directly reflected in the average distance of the $\mathrm{H}$-bond in the complex ${ }^{1} \mathrm{Ph}_{2} \mathrm{C} \cdots \mathrm{HOMe}$ (inset in Fig. 1). Remarkably, the lowest energy for ${ }^{1} \mathrm{Ph}_{2} \mathrm{C}$ is not found in neat $\mathrm{MeOH}$, but at very low concentrations of $\mathrm{MeOH}$. This suggests that ${ }^{1} \mathrm{Ph}_{2} \mathrm{C}$ is less stabilized if the $\mathrm{MeOH}$ molecule interacting with the carbene centre is also involved in an H-bonding network with other neighbouring methanol molecules. A series of QM/MM molecular dynamics (MD) simulations also corroborated that ${ }^{1} \mathrm{Ph}_{2} \mathrm{C}$ forms a hydrogen bonded complex in pure $\mathrm{MeOH}$, while ${ }^{3} \mathrm{Ph}_{2} \mathrm{C}$ does

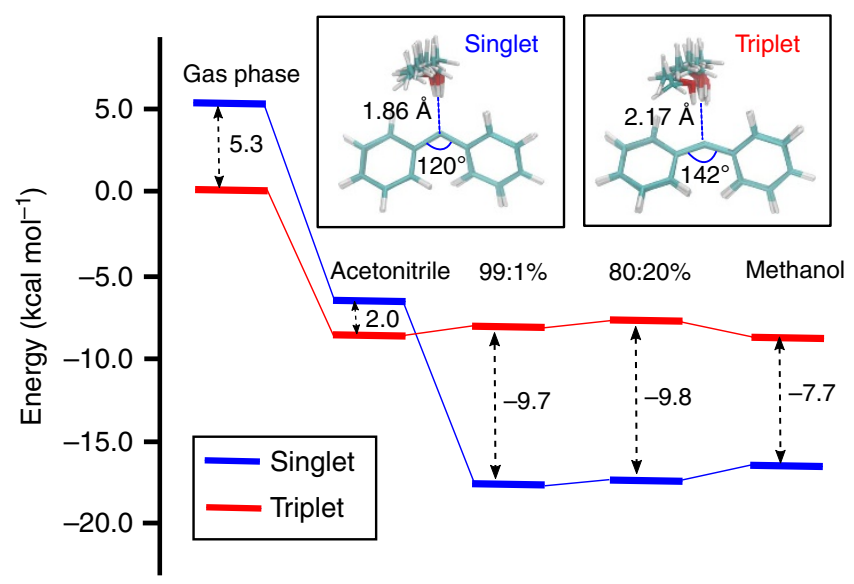

Figure 1 | Calculated singlet-triplet energy gaps of $\mathbf{P h}_{\mathbf{2}} \mathbf{C}$. The values ( $\mathrm{kcal} \mathrm{mol}{ }^{-1}$ ) correspond to $\mathrm{Ph}_{2} \mathrm{C}$ in the gas phase, pure solvents and different mixtures of $\mathrm{MeOH}$ and $\mathrm{MeCN}$. The experimental gap of $\mathrm{Ph}_{2} \mathrm{C}$ in acetonitrile is reported as $2.6 \mathrm{kcal} \mathrm{mol}^{-1}$ by Eisenthal et al. ${ }^{5}$ The insets show the $\mathrm{H}$-bonded complexes of $\mathrm{MeOH}$ with ${ }^{1} \mathrm{Ph}_{2} \mathrm{C}$ and ${ }^{3} \mathrm{Ph}_{2} \mathrm{C}$. 
not (Supplementary Fig. 5). Such complexes between ${ }^{1} \mathrm{Ph}_{2} \mathrm{C}$ and $\mathrm{MeOH}$ are also observed in solvent mixtures with lower $\mathrm{MeOH}$ content, but the complex formation strongly depends on the diffusion of methanol molecules to approach the carbene centre (Supplementary Figs 6 and 7 and Supplementary Note 4).

On an ultrafast time scale, ${ }^{1} \mathrm{Ph}_{2} \mathrm{C}$ reacts with an adjacent $\mathrm{MeOH}$ molecule. Further simulations unveil that the reaction leading to $\mathrm{O}-\mathrm{H}$ insertion always starts with the protonation of ${ }^{1} \mathrm{Ph}_{2} \mathrm{C}$, but two different mechanisms are found. In mechanism 1 (Fig. 2a), H-bond formation with a $\mathrm{MeOH}$ molecule is almost instantly followed by transfer of a proton to the carbene to yield $\mathrm{Ph}_{2} \mathrm{CH}^{+}$, while the nascent methoxide ion takes up a proton from a neighbouring $\mathrm{MeOH}$ molecule. In this way, the two charges are spatially separated, and the initially $\mathrm{H}$-bonded $\mathrm{MeOH}$ molecule is not the one found in the final ether product. Instead, a different $\mathrm{MeOH}$ molecule later on transfers a proton to the methoxide ion, and combination of this newly formed methoxide with $\mathrm{Ph}_{2} \mathrm{CH}^{+}$results in the formation of the ether. In mechanism 2, the proton transfer occurs within the ${ }^{1} \mathrm{Ph}_{2} \mathrm{C} \cdots \mathrm{HOMe}$ complex, and the resulting methoxide anion combines with $\mathrm{Ph}_{2} \mathrm{CH}^{+}$to the final product. The protonation step is facilitated by a second $\mathrm{MeOH}$ molecule, which does not directly interact with the carbene (Fig. 2b). From the ten computed trajectories corresponding to ${ }^{1} \mathrm{Ph}_{2} \mathrm{C}$ in pure $\mathrm{MeOH}$, four followed mechanism 1 and five followed mechanism 2. One trajectory resulted in the formation of a side product (addition of methoxide to the phenyl ring, forming the $\sigma$-complex of a nucleophilic aromatic substitution reaction).

Concerning mechanism 2, it is worth mentioning that after the protonation step, a reversible proton transfer from the neighbouring $\mathrm{MeOH}$ to the methanol in the ${ }^{1} \mathrm{Ph}_{2} \mathrm{C} \cdots \mathrm{HOMe}$ complex was observed in one of the five trajectories. Unlike in the other four trajectories, where $\mathrm{Ph}_{2} \mathrm{CH}^{+}$instantaneously reacted
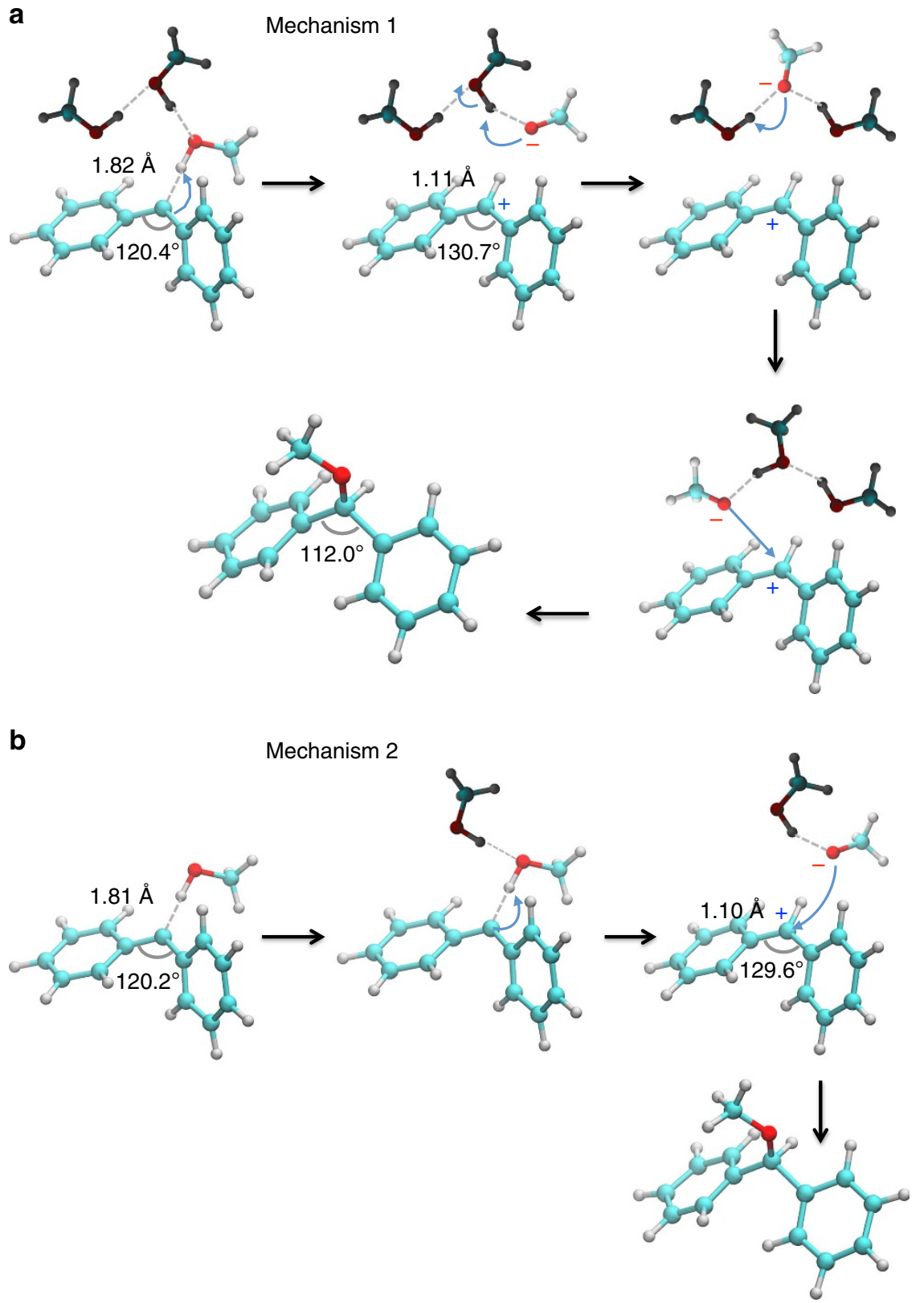

Figure 2 | Mechanisms 1 and $\mathbf{2}$ of $\mathbf{O}-\mathbf{H}$ insertion for the reaction of ${ }^{\mathbf{1}} \mathbf{P h}_{\mathbf{2}} \mathbf{C}$ with methanol. (a) Mechanism $1,(\mathbf{b})$ Mechanism 2. Average $\mathrm{OH} \cdots \mathrm{C}_{1 \mathrm{Ph} 2 \mathrm{C}}$ distances and $\mathrm{CCC}_{1 \mathrm{Ph} 2 \mathrm{C}}$ angles are shown. 
with the methoxide anion to form the insertion product, this reversible proton transfer contributed to stabilize the benzhydryl cation.

It should also be noted that in neat methanol, the second solvent molecule depicted in mechanism 2 is found already interacting with the methanol molecule hydrogen bonded to ${ }^{1} \mathrm{Ph}_{2} \mathrm{C}$. On the other hand, this is not necessarily the case in the solvent mixtures. There, it also happens that the second methanol diffuses after hydrogen bond formation towards the ${ }^{1} \mathrm{Ph}_{2} \mathrm{C} \cdots$ HOMe complex to facilitate the protonation reaction (Fig. 2b). In both cases the protonation reaction does not occur without a second $\mathrm{MeOH}$ molecule in the vicinity (see Supplementary Fig. 8 and Supplementary Note 5). This observation clearly suggests that a pathway involving the reaction of ${ }^{1} \mathrm{Ph}_{2} \mathrm{C}$ with a single $\mathrm{H}$-bonded $\mathrm{MeOH}$ molecule is disfavoured.

Experimental studies. Complementing our simulations, broadband transient absorption (TA) measurements on $\mathrm{Ph}_{2} \mathrm{CN}_{2}$ in solvent mixtures of $\mathrm{MeOH}$ and $\mathrm{MeCN}$ were conducted (see Methods) and intermediate species were identified by characteristic absorption signals. An extract of these measurements is presented in Fig. 3, a movie of all measurements is available as Supplementary Information. In the case of neat methanol (top left panel), two distinct positive TA signals are observed, which correspond to the absorption of ${ }^{1} \mathrm{Ph}_{2} \mathrm{C}$ centred at $355 \mathrm{~nm}$ and of the benzhydryl cation $\mathrm{Ph}_{2} \mathrm{CH}^{+}$at $435 \mathrm{~nm}$, respectively. The ground state bleach of the precursor $\mathrm{Ph}_{2} \mathrm{CN}_{2}$ appears on the high-energy edge of the detection range as negative TA signal. The spectral positions agree both with Kohler's study ${ }^{15}$ and with reported matrix experiments ${ }^{30,31}$ for $\mathrm{Ph}_{2} \mathrm{CH}^{+}$. Furthermore, they agree with recent measurements of the Riedle group on the photoinitiated carbocation formation from benzhydryl chlorides ${ }^{33-35}$. Additionally, and independent of the solvent mixing ratio, we observe the spectral signature of the excited diazo compound $\mathrm{Ph}_{2} \mathrm{CN}_{2}{ }^{*}$ at around $335 \mathrm{~nm}$ (Supplementary Fig. 9 and Supplementary Note 6), which decays with a time constant of $150 \mathrm{fs}$. For MeCN fractions above $90 \%$, the ground state bleach signal is completely overlaid by the positive TA contributions of the reaction intermediates.

The competition between different reaction pathways becomes evident from the TA data in solvent mixtures, where an increase in $\mathrm{MeCN}$ concentration leads to several changes in the dynamics: (1) the characteristic time scales increase, so ${ }^{1} \mathrm{Ph}_{2} \mathrm{C}$ decays more slowly, while $\mathrm{Ph}_{2} \mathrm{CH}^{+}$is formed later and survives for a longer time (see Fig. 3 and rate constants displayed in Fig. 4a). This observation confirms that reactions of the initially formed ${ }^{1} \mathrm{Ph}_{2} \mathrm{C}$ depend on the $\mathrm{MeOH}$ concentration, and so does the formation and decay of $\mathrm{Ph}_{2} \mathrm{CH}^{+} ;$(2) the decay rate of ${ }^{1} \mathrm{Ph}_{2} \mathrm{C}$ is always lower than the rate describing the rise of $\mathrm{Ph}_{2} \mathrm{CH}^{+}$. Hence, the absorption centred at $355 \mathrm{~nm}$ cannot originate solely from a precursor of $\mathrm{Ph}_{2} \mathrm{CH}^{+}$, but must also be due to a species which can co-exist with $\mathrm{Ph}_{2} \mathrm{CH}^{+}$in solution and which has a similar electronic absorption as ${ }^{1} \mathrm{Ph}_{2} \mathrm{C}$. We, therefore, conclude that the TA signal at $355 \mathrm{~nm}$ stems both from ${ }^{1} \mathrm{Ph}_{2} \mathrm{C}$ and the complex ${ }^{1} \mathrm{Ph}_{2} \mathrm{C} \cdots \mathrm{HOMe}$. This conclusion is supported by our NEVPT2/CASSCF calculations of the ultraviolet-visible transitions of ${ }^{1} \mathrm{Ph}_{2} \mathrm{C}$ and its complexes with water and $\mathrm{MeOH}$ (see Supplementary Fig. 10, Supplementary Table 5, and Supplementary Note 7 for more details); (3) the $\mathrm{Ph}_{2} \mathrm{CH}^{+}$ absorption becomes weaker compared to the initial ${ }^{1} \mathrm{Ph}_{2} \mathrm{C}$ signal (see for example, the panel for $80 \% \mathrm{MeCN}$ in Fig. 3). This directly shows that for reduced $\mathrm{MeOH}$ concentrations, $\mathrm{Ph}_{2} \mathrm{CH}^{+}$is less likely to be formed compared with ${ }^{1} \mathrm{Ph}_{2} \mathrm{C} \cdots \mathrm{HOMe}$; (4) for low $\mathrm{MeOH}$ fractions, the $\mathrm{Ph}_{2} \mathrm{CH}^{+}$signal disappears and a new absorption around $315 \mathrm{~nm}$ is observed after several hundred picoseconds (lower panels in Fig. 3). At very low concentrations of $\mathrm{MeOH}$, the ISC pathway to ${ }^{3} \mathrm{Ph}_{2} \mathrm{C}$ absorbing at $315 \mathrm{~nm}^{15,25}$ becomes dominant.

Additional information on solvation effects is deduced from the ultrafast dynamics in the solvent mixtures. Figure $4 \mathrm{~b}$ displays the temporal evolution of the maximum absorption wavelength of the $\mathrm{Ph}_{2} \mathrm{CH}^{+}$intermediate as derived from the TA data. For all mixtures with a sufficient amount of $\mathrm{Ph}_{2} \mathrm{CH}^{+}$, the starting position is at lower wavelengths for lower $\mathrm{MeOH}$ fractions, as a consequence of the solvent polarity ${ }^{36}$ and the degree of solvation
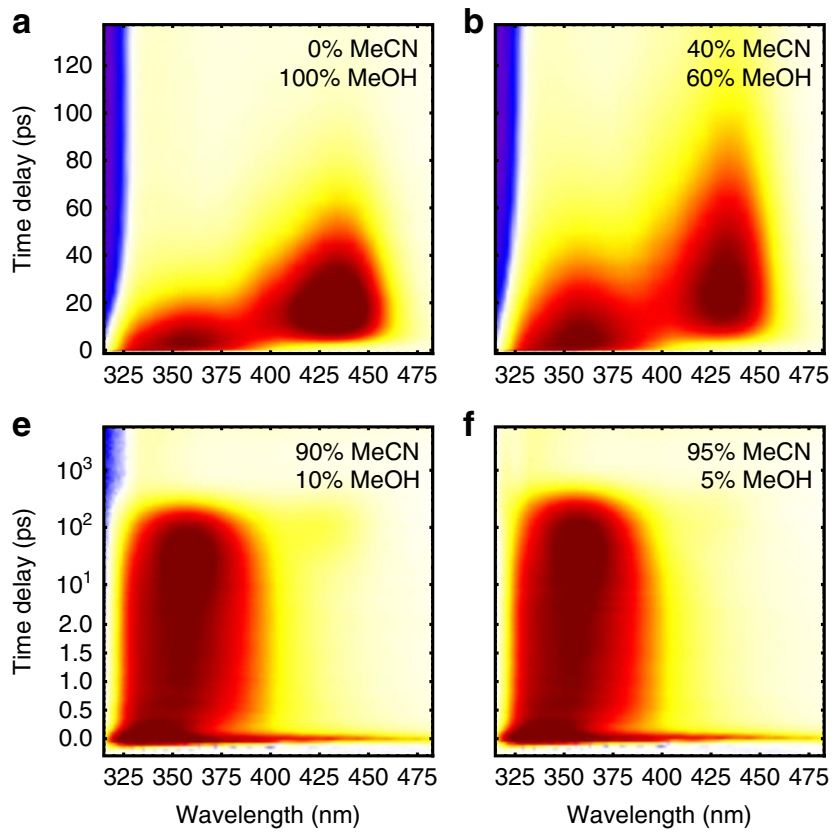
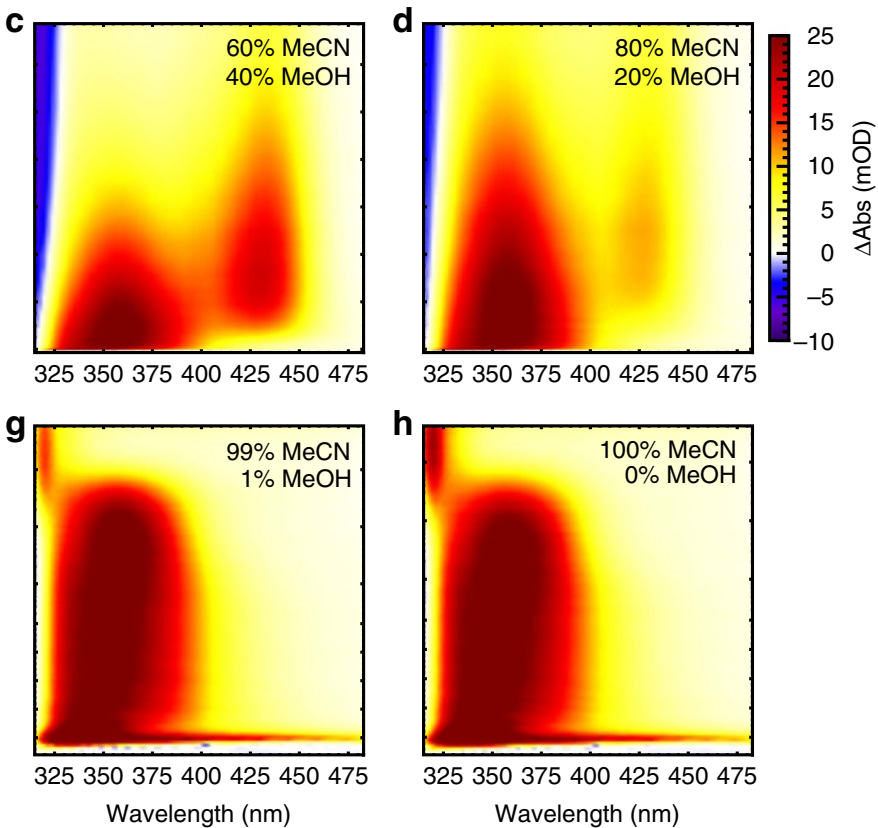

Figure 3 | Transient absorption of $\mathbf{P h}_{\mathbf{2}} \mathbf{C N} \mathbf{2}$ under $\mathbf{2 8 5} \mathbf{~ n m}$ excitation. Note that for concentrations up to $80 \%$ MeCN (a-d), data is shown using a linear time axis covering the first 135 ps pump-probe delay, whereas for higher MeCN concentrations (e-h) a lin-log time axis is chosen in order to include dynamics up to $4 \mathrm{~ns}$ pump-probe delay. 
a

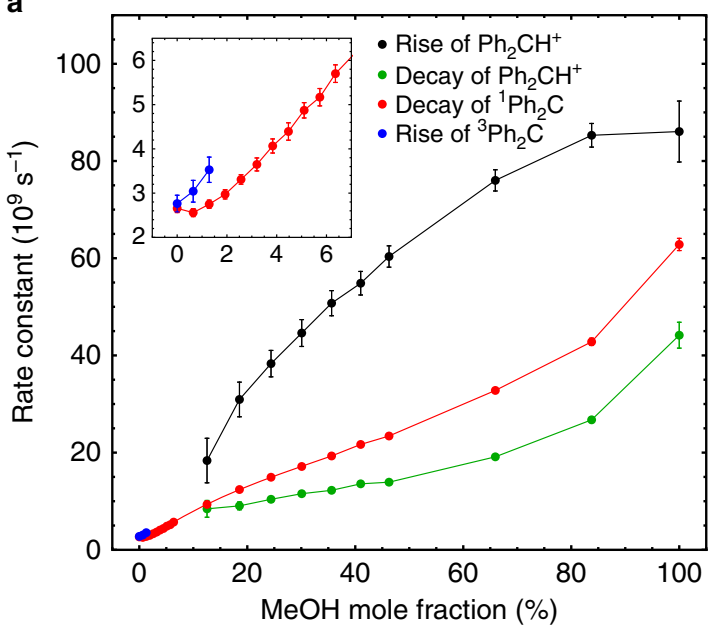

b

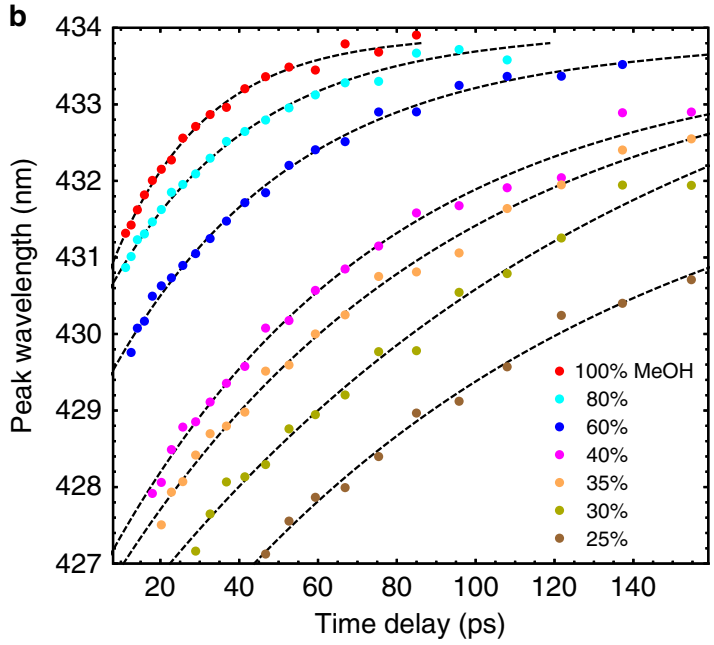

Figure 4 | Effects of solvent mixing ratio on reaction dynamics. (a) Rate constants extracted from nonlinear interpolation describing the rise and the decay of $\mathrm{Ph}_{2} \mathrm{CH}^{+}$(maximum wavelength), the decay of ${ }^{1} \mathrm{Ph}_{2} \mathrm{C}(355 \mathrm{~nm})$, as well as the rise of ${ }^{3} \mathrm{Ph}_{2} \mathrm{C}(315 \mathrm{~nm})$ in solvent mixtures of varying MeOH mole fractions. The bars indicate $95 \%$ confidence intervals ( \pm 1.96 s.d.). (b) Time-dependent peak position of the $\mathrm{Ph}_{2} \mathrm{CH}^{+}$absorption band. For all solvent mixtures, a red-shift is observed, but the initial wavelength increases with $\mathrm{MeOH}$ volume fraction. See Supplementary Tables 6-9 for the data, Supplementary Figs 11-13 and Supplementary Note 6 for details on analysis and fitting.

of the precursor ${ }^{1} \mathrm{Ph}_{2} \mathrm{C}$ in the different mixtures. Furthermore, a red-shift of the $\mathrm{Ph}_{2} \mathrm{CH}^{+}$signal is observed, which originates from initial solvation of the cation, but after several picoseconds mostly reflects intramolecular geometrical changes and the separation of the benzhydryl cation and the methoxide anion. This separation leads to a shrinking energy gap between the ground and excited states of the benzhydryl cation, as shown by Riedle and coworkers in studies on benzhydryl halides ${ }^{35}$. Hence, the simultaneously generated methoxide anion does not geminately react with $\mathrm{Ph}_{2} \mathrm{CH}^{+}$, which thereby becomes observable in the experiments. While diffusion may contribute, we propose that the separation process involves a second $\mathrm{MeOH}$ molecule, as suggested in refs 12,26 and also confirmed by our simulations. For the singlet carbene absorption (see Supplementary Fig. 14 and Supplementary Note 6), we observe a red-shift during the first ten ps. The solvent environment adjusted to the diazo precursor has to rearrange upon the formation of ${ }^{1} \mathrm{Ph}_{2} \mathrm{C}$, and this solvent rearrangement is reflected in the spectral shift which for carbenes is bathochromic ${ }^{17}$

The experiments described above allow us to estimate the amount of molecules following the different reaction pathways. Starting out with the dynamics in neat $\mathrm{MeOH}$, a fraction $x$ of the initial ${ }^{\mathrm{P}} \mathrm{Ph}_{2} \mathrm{C}$ molecules takes up a proton to form $\mathrm{Ph}_{2} \mathrm{CH}^{+}$. This charged species then rapidly reacts on to an ether which is dark (that is, non-absorbing) in the accessible spectral range. Kohler and coworkers ${ }^{15}$ showed that about $30 \%$ of the photoexcited $\mathrm{Ph}_{2} \mathrm{CN}_{2}$ molecules lead to $\mathrm{Ph}_{2} \mathrm{CH}^{+}$, but an assignment of whether the other $70 \%$ do not lead to the formation of ${ }^{1} \mathrm{Ph}_{2} \mathrm{C}$ (that is, $x=1$ ) or follow an alternative reaction pathway to the dark product (that is, $x=0.3$ ) could not be made. We conclude the latter scenario occurs as for all mixtures and even in neat $\mathrm{MeOH}$ (Fig. 4a) the decay rate for the singlet carbene is lower than the rise of $\mathrm{Ph}_{2} \mathrm{CH}^{+}$, corroborating ${ }^{1} \mathrm{Ph}_{2} \mathrm{C} \cdots \mathrm{HOMe}$ formation. Hence, $x$ must be smaller than one but in principle could also be larger than 0.3. A direct comparison of the TA data for different mixtures is possible after normalization to the initial ${ }^{1} \mathrm{Ph}_{2} \mathrm{C}$ signal and taking into account the two extreme cases, that is, that in neat $\mathrm{MeOH} 30 \%$ react via $\mathrm{Ph}_{2} \mathrm{CH}^{+}$, whereas in pure MeCN $100 \%$ form ${ }^{3} \mathrm{Ph}_{2} \mathrm{C}$. The latter assumption is evidenced by the matching decay rate of ${ }^{1} \mathrm{Ph}_{2} \mathrm{C}$ and rise rate of ${ }^{3} \mathrm{Ph}_{2} \mathrm{C}$ in Fig. 4a and the absence of other singlet decay channels in pure $\mathrm{MeCN}$ (ref. 15). The estimated fractions of molecules following the benzhydryl cation and the triplet pathways are displayed in Fig. 5a; the derivation of these results from the TA data is given in the Supplementary Note 8 and Supplementary Table 10. The fact that both fractions do not add up to one (independent of the value $x$ ) confirms the existence of a third channel (occurring more frequently in solvent mixtures) attributed to ${ }^{1} \mathrm{Ph}_{2} \mathrm{C} \cdots \mathrm{HOMe}$. The red data points in Fig. 5a correspond to the amount of molecules following this pathway, as indirectly deduced from the other fractions.

The results from the TA experiments allow us to construct a reasonable reaction scheme, which furthermore is in full accordance with the simulated mechanisms and previous studies in cryogenic matrices ${ }^{30}$. The separation of cation and anion indicates that more than one $\mathrm{MeOH}$ molecule is involved in the $\mathrm{Ph}_{2} \mathrm{CH}^{+}$formation. This is included in the scheme as the simple case of a quadratic dependence on the $\mathrm{MeOH}$ concentration. Furthermore, the pathway via the complex ${ }^{1} \mathrm{Ph}_{2} \mathrm{C} \cdots \mathrm{HOMe}$ was found, which depends linearly on the $\mathrm{MeOH}$ concentration. Both of these reactions lead to the ether as final product, but for the final step a further molecule of $\mathrm{MeOH}$ is required. The third pathway is the ISC to ${ }^{3} \mathrm{Ph}_{2} \mathrm{C}$, which contributes more at lower $\mathrm{MeOH}$ fractions. A reaction scheme comprising all of these aspects is sketched in Fig. 5b. An analytical solution describing the associated dynamics is given in the Supplementary Note 9 and Supplementary Figs 15-17, together with a discussion of why simpler models cannot reliably describe the experimental data.

To model the product channels shown in Fig. 5 using this reaction scheme, the corresponding three rate constants connected to the depopulation of ${ }^{1} \mathrm{Ph}_{2} \mathrm{C}$ are required. The ISC rate constant can be calculated from literature data by combining the polarity dependence of this rate ${ }^{11}$ with the polarity of the solvent mixtures ${ }^{36}$. The other two are estimated from the experimental decay rate of ${ }^{1} \mathrm{Ph}_{2} \mathrm{C}$ in pure $\mathrm{MeOH}$ (see Supplementary Note 6 for fitting procedure) and the assumption $x=0.3$. Note that no other parameter, in particular no free parameter is needed. This reaction scheme accurately describes the experimental data (solid lines in Fig. 5a), and confirms that there is a solvent mixing ratio for which the amount of molecules reacting via the ${ }^{1} \mathrm{Ph}_{2} \mathrm{C} \cdots$ HOMe complex reaches a maximum. 
a

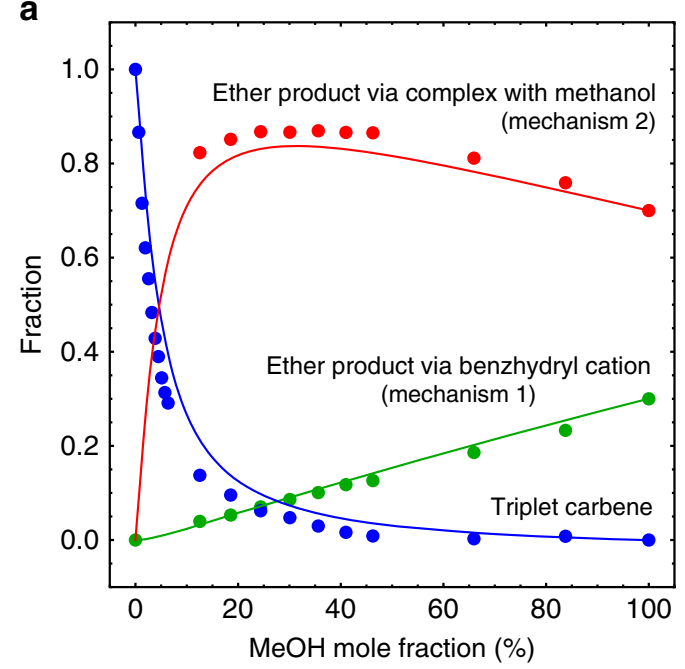

b

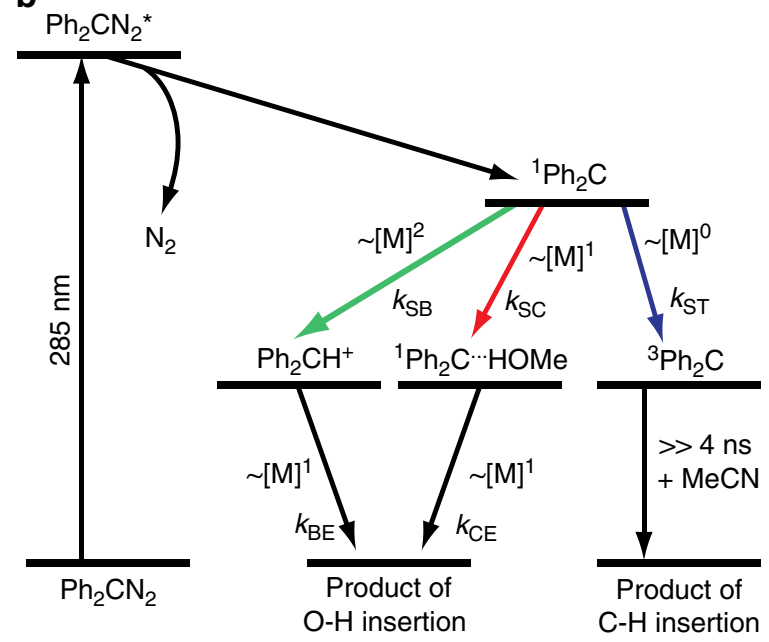

Figure 5 | Analysis of reaction channels. (a) Fraction of molecules following the $\mathrm{Ph}_{2} \mathrm{CH}^{+}$(green), the ${ }^{3} \mathrm{Ph}_{2} \mathrm{C}$ (blue) and the ${ }^{1} \mathrm{Ph}_{2} \mathrm{C} \cdots \mathrm{HOMe}($ red) pathway for different $\mathrm{MeOH} / \mathrm{MeCN}$ solvent mixtures, as derived from the TA data. The solid curves result from the reaction scheme sketched in $\mathbf{b}$, where the rates exhibit different dependencies on the $\mathrm{MeOH}$ concentration [M]

Eisenthal and coworkers ${ }^{8,9}$ found by comparing product ratios that, for ${ }^{1} \mathrm{Ph}_{2} \mathrm{C}$ in aprotic solvents with small amounts of $\mathrm{MeOH}$ cosolvent, the alcohol exerts a 'twofold effect': on the one hand stabilizing the singlet carbene and on the other hand opening a decay channel via the concerted $\mathrm{O}-\mathrm{H}$ insertion. Our TA data discloses this behavior in a straightforward way via the decay rate of the singlet carbene: the lowest value is not observed for pure $\mathrm{MeCN}$ but around $99.5 \%$ (inset of Fig. 4a), directly confirming that single $\mathrm{MeOH}$ molecules have a stabilizing effect on ${ }^{1} \mathrm{Ph}_{2} \mathrm{C}$. Beyond that, these room-temperature studies in solution nicely complement the argon matrix experiments at $3 \mathrm{~K}$ doped with $1 \%$ $\mathrm{MeOH}$ in which also the ${ }^{1} \mathrm{Ph}_{2} \mathrm{C} \cdots \mathrm{HOMe}$ complex is formed by reaction of $\mathrm{Ph}_{2} \mathrm{C}$ with single molecules of $\mathrm{MeOH}$, eventually yielding the ether product in a tunnelling process ${ }^{30}$.

\section{Discussion}

Our combined experimental and theoretical results provide a clear and conclusive picture of the fate of ${ }^{1} \mathrm{Ph}_{2} \mathrm{C}$ formed photochemically in solution, thereby considering three competing reaction channels with different dependencies on the $\mathrm{MeOH}$ concentration. On the one hand, at low $\mathrm{MeOH}$ concentrations, ${ }^{1} \mathrm{Ph}_{2} \mathrm{C}$ can form an $\mathrm{H}$-bonded complex with a $\mathrm{MeOH}$ molecule. Subsequently, after a second $\mathrm{MeOH}$ molecule approaches, the ether product can be formed according to mechanism 2. Since the same $\mathrm{MeOH}$ molecule transfers the proton and completes the reaction, signatures of charged intermediates are extremely short-lived, if observable at all. Unlike in the solvent mixtures, in pure methanol a second solvent molecule can be easily found interacting with the hydrogen-bonded methanol. Our analysis suggests that mechanism 2 is the dominant mechanism in neat $\mathrm{MeOH}$ (see Fig. 5). On the other hand, as ${ }^{1} \mathrm{Ph}_{2} \mathrm{C}$ encounters a $\mathrm{MeOH}$ molecule that already interacts with one (or more) neighbouring $\mathrm{MeOH}$, mechanisms 1 or 2 can take place. Due to the separation of $\mathrm{Ph}_{2} \mathrm{CH}^{+}$and the methoxide, the charged species produced by mechanism 1 survive for a longer time, and $\mathrm{Ph}_{2} \mathrm{CH}^{+}$is identifiable by its characteristic absorption.

Concluding, our results permit two main inferences: singlet diphenylcarbene ${ }^{1} \mathrm{Ph}_{2} \mathrm{C}$, formed on a femtosecond time scale in binary solvent mixtures $(\mathrm{MeOH} / \mathrm{MeCN})$, is subject to three reaction mechanisms depending on the number of $\mathrm{MeOH}$ molecules that this reactive species initially encounters: (i) ISC to ${ }^{3} \mathrm{Ph}_{2} \mathrm{C}$, (ii) formation of an $\mathrm{H}$-bonded complex ${ }^{1} \mathrm{Ph}_{2} \mathrm{C} \cdots \mathrm{HOMe}$, which reacts on to the ether product in a concerted fashion activated by a further $\mathrm{MeOH}$ molecule or (iii) ether production via an intermediate $\mathrm{Ph}_{2} \mathrm{CH}^{+}$, which requires that two $\mathrm{MeOH}$ molecules interact concurrently with ${ }^{1} \mathrm{Ph}_{2} \mathrm{C}$. Additionally, the concentration of 'available' molecules of $\mathrm{MeOH}$, that is, molecules that can hydrogen bond with ${ }^{1} \mathrm{Ph}_{2} \mathrm{C}$ or facilitate the reaction via $\mathrm{MeOH}-\mathrm{MeOH}$ interactions, rather than the absolute concentration of $\mathrm{MeOH}$ in $\mathrm{MeCN}$ solutions, determines the reaction pathway, which is in striking analogy with results from matrix isolation experiments at cryogenic temperatures ${ }^{30}$. Hence, variation of the solvent mixing ratio provides a means not only to control the time scales of the reaction, but also the reactivity, that is, which reaction path is followed.

In more general terms, even if an intermediate in solution may formally just react with a single solvent molecule, further, not directly involved solvent molecules may tip the scales in favour of a certain reaction mechanism. This nonlinear behaviour is not limited to binary solvent mixtures, and is of general importance for all intermediates capable of reacting with solvents. Our study demonstrates that solvent mixtures, if understood correctly, are versatile tools to control chemical reactivity.

\section{Methods}

Simulations. The reactivity of diphenylcarbene $\left(\mathrm{Ph}_{2} \mathrm{C}\right)$ and the relative stabilities of its singlet $\left({ }^{1} \mathrm{Ph}_{2} \mathrm{C}\right)$ and triplet $\left({ }^{3} \mathrm{Ph}_{2} \mathrm{C}\right)$ states in different solvent conditions, (I) acetonitrile (MeCN), (II) methanol (MeOH), (III) $\mathrm{MeCN} / \mathrm{MeOH}(80: 20 \% \mathrm{v} / \mathrm{v}$ mixture) and (IV) $\mathrm{MeCN} / \mathrm{MeOH}$ (99:1\% v/v mixture) were investigated using QM/MM calculations to capture the solvent effects and specific interactions regulating the chemistry of $\mathrm{Ph}_{2} \mathrm{C}$.

Force field parameterization. Classical force field parameters for $\mathrm{MeOH}$ and $\mathrm{MeCN}$ are available in the OPLS all-atom force field, which is able to reproduce the physical properties of these solvents in liquid simulations ${ }^{37}$. Since, currently, the OPLS force field is not supported by the ChemShell code ${ }^{38,39}$, the OPLS parameters of $\mathrm{MeOH}$ and $\mathrm{MeCN}$ were fitted to the CHARMM force field format. OPLS parameterization requires scaling-down of 1-4 non-bonded interactions (both van der Waals and Coulomb interactions) by $50 \%$, in contrast to full scaling in CHARMM. The non-bonded interaction, bond stretching and bond-angle bending parameters were used as such ${ }^{37}$. The dihedral angle parameters were modified to compensate for the full 1-4 scaling. The dihedral angle parameters were optimized to match the energy difference between eclipsed and staggered conformations (calculated at the $\mathrm{HF} / 6-31 \mathrm{G}^{*}$ level). The force field parameters for the solvents used in this study are given in Supplementary Table 1. The new parameters were validated by the liquid properties obtained from MD simulations. 
$\mathrm{Ph}_{2} \mathrm{C}$ was also parameterized for preliminary classical MD simulations. The initial parameters were obtained using the SwissParam webserver (www.swissparam.ch/ $/)^{40}$ and the partial atomic charges were calculated by fitting the restrained electrostatic potential (RESP) charges calculated at the HF/6-31G* level. The RESP charge derive (RED) webserver (http://q4md-forcefieldtools.org/ $\mathrm{RED} /$ ) was used for this purpose ${ }^{41-43}$

Classical MD simulations. ${ }^{1} \mathrm{Ph}_{2} \mathrm{C}$ was calculated under four solvent conditions, (I) $\mathrm{MeCN}$, (II) $\mathrm{MeOH}$, (III) $\mathrm{MeCN} / \mathrm{MeOH}(80: 20 \% \mathrm{v} / \mathrm{v}$ mixture) and (IV) $\mathrm{MeCN} / \mathrm{MeOH}$ (99:1\% v/v mixture). Set-ups I, II and III were simulated for $5 \mathrm{~ns}$ in a cubic periodic box at $300 \mathrm{~K}$ and $1 \mathrm{~atm}$ pressure, using $2 \mathrm{fs}$ as timestep. Set-up IV was simulated for $20 \mathrm{~ns}$ under the same conditions. The cutoff for short-range non-bonded interactions was $10 \AA$. The non-bonded potential was smoothly shifted to zero at $12 \AA$. Particle mesh Ewald summation was used to account for long-range electrostatics. $\mathrm{Ph}_{2} \mathrm{C}$ was kept rigid. All the classical MD simulations were carried out using the nanoscale molecular dynamics (NAMD) program ${ }^{44}$.

QM/MM MD simulations. Snapshots of $\mathrm{Ph}_{2} \mathrm{C}$ in a droplet of solvent (of $30 \AA$ radius from the carbene center) were taken from the classical MD trajectories. Then, $\mathrm{Ph}_{2} \mathrm{C}$ in the solvent sphere was subjected to $10 \mathrm{ps} \mathrm{QM} / \mathrm{MM}$ MD simulation, at $300 \mathrm{~K}$ with positional restraints on the carbene center. The QM region was formed by the $\mathrm{Ph}_{2} \mathrm{C}$ molecule and treated at the B3LYP-D3/def2-SVP level of theory ${ }^{4-48}$ while the solvents were treated at the MM level (see reactivity studies section for larger QM regions). The ChemShell package was used for all the QM/MM calculations, with Turbomole (v6.6) for the QM region ${ }^{49}$ and DL-POLY code $^{50}$ for the MM part. The interactions between QM and MM subsystems were calculated using an electrostatic embedding scheme, in which the MM point charges polarize the QM charge density ${ }^{51}$.

QM/MM optimizations. Ten snapshots taken from the last $2 \mathrm{ps}$ of the QM/MM MD simulations were used for QM/MM optimizations. During the QM/MM optimization, $\mathrm{Ph}_{2} \mathrm{C}$ was treated at the B3LYP-D3/def2-TZVPP level ${ }^{52}$. QM/MM MD simulations and optimizations were performed separately for singlet and triplet states.

Synthesis of precursor. Benzophenone (Sigma Aldrich, 99\%) and p-Toluenesulfonyl hydrazide (ABCR, 98\%) were used without further purification. Benzophenone tosylhydrazone was prepared according to a literature procedure ${ }^{53}$ by refluxing for $40 \mathrm{~h}$ a mixture of 1 eq. of benzophenone and 2 eq. of p-toluenesulfonyl hydrazide in absolute ethanol. The crude product was purified by recrystallization in ethanol. By treating the tosylhydrazone with 1.1 eq. of $\mathrm{NaH}$ ( $60 \%$ dispersion in mineral oil) in dry $\mathrm{CH}_{2} \mathrm{Cl}_{2}$ the corresponding sodium salt is formed. Sublimation of the salt on a cold substrate at $45^{\circ} \mathrm{C}$ under reduced pressure yields the characteristic dark purple diphenyldiazomethane. Infrared (Ar, $3 \mathrm{~K}): 3,070(\mathrm{~m})$, 2,046 (vs), 1,598 (s), 1,582 (m), 1,502 (s), 1,497 (s), 1,457 (m), 1,447 (m), 1,320 (m), 1,268 (m), 1,262 (m), 1,034 (m), $936(\mathrm{~m}), 756(\mathrm{~s}), 750(\mathrm{~s}), 697(\mathrm{~s}), 692(\mathrm{~s}), 651(\mathrm{~s})$, $482(\mathrm{~m}) \mathrm{cm}^{-1}$. H NMR (200 MHz, DMSO) $\delta=7.44(\mathrm{~m}, 4 \mathrm{H}), 7.27(\mathrm{~m}, 6 \mathrm{H})$.

Sample preparation. Even small fluctuations in the sample concentration would lead to changes in the absolute absorption-change signals and, therefore, affect the comparison of quantitative values such as the amount of $\mathrm{Ph}_{2} \mathrm{CH}^{+}$produced in different solvent environments. Hence, we use a $\mathrm{MeOH}$ and a $\mathrm{MeCN}$ parent solution, respectively, each containing $294 \mathrm{mg} \mathrm{Ph}_{2} \mathrm{CN}_{2}$ in $13 \mathrm{ml}$ solvent. Despite the high concentration in the latter solutions, no problems regarding solubility have been observed. For each $20 \mathrm{ml}$ sample solution, $1 \mathrm{ml}$ of the corresponding parent solution was extracted using a volumetric pipette. Which parent solution is taken is determined by the desired solvent ratio. In our case, concentrations between 0 to $90 \%$ $\mathrm{MeCN}$ were prepared using the $\mathrm{MeOH}$ parent solution, whereas the $\mathrm{MeCN}$ parent solution was the starting point for solutions containing 95 to $100 \% \mathrm{MeCN}$. To finally obtain the sample solutions under investigation, missing solvent was separately extracted using volumetric pipettes and added to the $1 \mathrm{ml}$ of parent solution.

Solvents (Merck Millipore, Uvasol) were used as received. All samples examined were prepared for equal molarity although the molar masses of $\mathrm{MeOH}$ and $\mathrm{MeCN}$ significantly differ. Albeit both solvents have approximately the same density, the ratio of the actual number of solvent molecules of each sort differs from the associated volume ratio. Proper sample exchange was ensured by cleaning the tubing system after recording each data set with a pure solvent mixture, which corresponded to the subsequently examined sample mixture.

TA measurements. The TA of diphenylcarbene $\left(\mathrm{Ph}_{2} \mathrm{C}\right)$ generated via ultraviolet photolysis of the diazo-compound precursor diphenyldiazomethane $\left(\mathrm{Ph}_{2} \mathrm{CN}_{2}\right)$, is reported in solvent mixtures of $\mathrm{MeOH}$ and $\mathrm{MeCN}$. All radiation employed originates from a regenerative Ti:Sapphire amplifier system (Solstice; Spectra Physics: $1 \mathrm{kHz}, 800 \mathrm{~nm}, 100 \mathrm{fs}$ ). The latter is used to pump a non-collinear optical parametric amplifier (TOPAS-White; Light Conversion, Ltd) in order to generate $285 \mathrm{~nm}$ pump pulses. These pulses are subsequently compressed using an acousto-optic programmable dispersive filter (Dazzler; Fastlite) ${ }^{54}$ to a pulse duration of $40 \mathrm{fs}$ at the sample position, as determined by difference-frequency cross-correlation frequency-resolved optical gating (XFROG) measurements ${ }^{55,56}$.
Another fraction of the $800 \mathrm{~nm}$ fundamental beam is focused into a linearly moving $\mathrm{CaF}_{2}$ plate to generate supercontinuum probe pulses with spectral components down to $\approx 300 \mathrm{~nm}$. The beams, focused to diameters of roughly $50 \mu \mathrm{m}$ (pump) and $40 \mu \mathrm{m}$ (probe), respectively, are spatially overlapped at the sample position, whereas the polarization directions are held at the magic angle configuration $\left(54.7^{\circ}\right)$ (ref. 57). For excitation, $130 \mathrm{~nJ}$ pump pulses are applied to stay in the linear excitation regime (Supplementary Fig. 18 and Supplementary Note 10), whereas the energy of the probe pulses is substantially lower. A spectrally resolved shot-to-shot detection of changes in optical density between the pumped and unpumped probe volume is enabled by using a visible spectrograph (Acton SP2500i; Princeton Instruments) combined with a two-dimensional CCD (charge-coupled device) camera (Pixis $2 \mathrm{~K}$; Princeton Instruments) with an acquisition rate of $1 \mathrm{kHz}$ and mechanically chopping the pump pulses at $500 \mathrm{~Hz}$. To exchange the probed sample volume between subsequent pump-probe pairs, a $20 \mathrm{ml}$ sample volume is perpetually pumped through a Suprasil flow cell of $200 \mu \mathrm{m}$ path length, whereby $6 \mathrm{mM}$ solutions of the precursor are employed, resulting in an optical density of about 2 at $285 \mathrm{~nm}$ along the flow cell. Measurement times are kept sufficiently short to prevent major sample degradation due to continuous photolysis of the precursor, as verified by measurements of the linear absorption spectrum before and after the time-resolved experiments. In the measurement routine, the pump-probe delay is scanned up to $4 \mathrm{~ns}$ using linear step sizes within the first few picoseconds followed by exponential step sizes, whereas the average of 1,000 consecutive TA spectra is recorded at each time delay. No mechanical changes in the set-up-alignment were made when recording TA data for a total of 21 sample solutions. All data shown results from averaging over three subsequently recorded data sets in order to minimize long-term fluctuations. The data has been corrected for the chirp of the supercontinuum probe ${ }^{57}$.

Data availability. Data supporting the findings of this study are available within the article and Supplementary Information, as well as from the corresponding authors upon reasonable request.

\section{References}

1. Kirmse, W. Carbene Chemistry (Academic Press Inc, 1971).

2. Wang, Y., Sitzmann, E. V., Novak, F., Dupuy, C. \& Eisenthal, K. B. Reactions of excited triplet diphenylcarbene studied with picosecond lasers. J. Am. Chem. Soc. 104, 3238-3239 (1982).

3. Sitzmann, E. V., Wang, Y. \& Eisenthal, K. B. Picosecond laser studies on the reaction of excited triplet diphenylcarbene with alcohols. J. Phys. Chem. 87, 2283-2285 (1983).

4. Sitzmann, E. V., Langan, J. \& Eisenthal, K. B. Picosecond laser studies of the charge-transfer reaction of excited triplet diphenylcarbene with electron donors. Chem. Phys. Lett. 102, 446-450 (1983)

5. Eisenthal, K. B., Moss, R. A. \& Turro, N. J. Divalent carbon intermediates: laser photolysis and spectroscopy. Science 225, 1439-1445 (1984).

6. Sitzmann, E. V., Langan, J. \& Eisenthal, K. B. Intermolecular effects on intersystem crossing studied on the picosecond timescale: the solvent polarity effect on the rate of singlet-to-triplet intersystem crossing of diphenylcarbene. $J$. Am. Chem. Soc. 106, 1868-1869 (1984).

7. Langan, J. G., Sitzmann, E. V. \& Eisenthal, K. B. Picosecond laser studies on the effect of structure and environment on intersystem crossing in aromatic carbenes. Chem. Phys. Lett. 110, 521-527 (1984).

8. Sitzmann, E. V., Langan, J. G. \& Eisenthal, K. B. Picosecond laser studies of the effects of reactants on intramolecular energy relaxation of diphenylcarbene: reaction of diphenylcarbene with alcohols. Chem. Phys. Lett. 112, 111-116 (1984)

9. Eisenthal, K. B. et al. Singlet-triplet interconversion of diphenylmethylene. Energetics, dynamics and reactivities of different spin states. Tetrahedron 41, 1543-1554 (1985).

10. Langan, J. G., Sitzmann, E. V. \& Eisenthal, K. B. Inverse deuterium isotope effect in the intersystem crossing of diphenylcarbene. Chem. Phys. Lett. 124, 59-62 (1986)

11. Sitzmann, E. V., Langan, J. G., Griller, D. \& Eisenthal, K. B. Effects of solvent polarity and structure on intersystem crossing in diphenylcarbenes. A picosecond laser study on dimesitylcarbene. Chem. Phys. Lett. 161, 353-360 (1989).

12. Kirmse, W., Guth, M. \& Steenken, S. Production of $\alpha$-siloxycarbenium ions by protonation of photochemically generated $\alpha$-siloxycarbenes. Formation mechanism and reactivities with nucleophiles. J. Am. Chem. Soc. 118, 10838-10849 (1996).

13. Sander, W., Bucher, G. \& Wierlacher, S. Carbenes in matrixes: spectroscopy, structure, and reactivity. Chem. Rev. 93, 1583-1621 (1993).

14. Portella-Oberli, M. T., Jeannin, C., Soep, B., Zerza, G. \& Chergui, M. Femtosecond study of the rise and decay of carbenes in solution. Chem. Phys. Lett. 296, 323-328 (1998).

15. Peon, J., Polshakov, D. \& Kohler, B. Solvent reorganization controls the rate of proton transfer from neat alcohol solvents to singlet diphenylcarbene. J. Am. Chem. Soc. 124, 6428-6438 (2002).

16. Showalter, B. M. \& Toscano, J. P. Time-resolved IR studies of $\alpha$-lactones. J. Phys. Org. Chem. 17, 743-748 (2004). 
17. Wang, J., Kubicki, J., Gustafson, T. L. \& Platz, M. S. The dynamics of carbene solvation: an ultrafast study of p-biphenylyltrifluoromethylcarbene. J. Am. Chem. Soc. 130, 2304-2313 (2008).

18. Wang, J., Zhang, Y., Kubicki, J. \& Platz, M. S. Ultrafast studies of some diarylcarbenes. Photochem. Photobiol. Sci. 7, 552 (2008).

19. Rudolf, P., Buback, J., Aulbach, J., Nuernberger, P. \& Brixner, T. Ultrafast multisequential photochemistry of 5-diazo Meldrum's acid. J. Am. Chem. Soc. 132, 15213-15222 (2010).

20. Burdzinski, G. \& Platz, M. S. Ultrafast time-resolved studies of the photochemistry of diazo carbonyl compounds. J. Phys. Org. Chem. 23, 308-314 (2010).

21. Steinbacher, A., Roeding, S., Brixner, T. \& Nuernberger, P. Ultrafast photofragment ion spectroscopy of the Wolff rearrangement in 5-diazo Meldrum's acid. Phys. Chem. Chem. Phys. 16, 7290 (2014).

22. Burdzinski, G. \& Platz, M. S. in Contemporary Carbene Chemistry. (eds Moss, R. A. \& Doyle, M. P.) 166-192 (Wiley, 2013).

23. Kirmse, W., Horner, L. \& Hoffmann, H. Über Lichtreaktionen IX. Umsetzungen photochemisch erzeugter Carbene. Liebigs Ann. Chem. 614, 19-30 (1958).

24. Griller, D., Nazran, A. S. \& Scaiano, J. C. Reaction of diphenylcarbene with methanol. J. Am. Chem. Soc. 106, 198-202 (1984).

25. Hadel, L. M., Platz, M. S. \& Scaiano, J. C. Study of hydrogen atom abstraction reactions of triplet diphenylcarbene in solution. J. Am. Chem. Soc. 106, 283-287 (1984).

26. Steenken, S. Production of carbenium ions from carbenes by protonation. Pure Appl. Chem. 70, 2031-2038 (1998).

27. Bethell, D., Newall, A. R., Stevens, G. \& Whittaker, D. Intermediates in the decomposition of aliphatic diazo-compounds. Part VII. Mechanisms for formation of benzophenone azine and diphenylmethanol in the thermal decomposition of diphenyldiazomethane. J. Chem. Soc. B 749-754 (1969).

28. Turro, N. J., Cha, Y. \& Gould, I. R. Temperature dependence of the reactions of singlet and triplet diphenylcarbene. Tetrahedron Lett. 26, 5951-5954 (1985).

29. Xue, J., Luk, H. L. \& Platz, M. S. Direct observation of a carbene-alcohol ylide. J. Am. Chem. Soc. 133, 1763-1765 (2011).

30. Costa, P. \& Sander, W. Hydrogen bonding switches the spin state of diphenylcarbene from triplet to singlet. Angew. Chem. Int. Ed. 53, 5122-5125 (2014).

31. Costa, P., Fernandez-Oliva, M., Sanchez-Garcia, E. \& Sander, W. The highly reactive benzhydryl cation isolated and stabilized in water ice. J. Am. Chem. Soc. 136, 15625-15630 (2014).

32. Marcus, Y. Preferential solvation in mixed solvents. Part 6. Binary mixtures containing methanol, ethanol, acetone or triethylamine and another organic solvent. J. Chem. Soc. Faraday Trans. 87, 1843 (1991).

33. Ammer, J., Sailer, C. F., Riedle, E. \& Mayr, H. Photolytic generation of benzhydryl cations and radicals from quaternary phosphonium salts: how highly reactive carbocations survive their first nanoseconds. J. Am. Chem. Soc. 134, 11481-11494 (2012).

34. Fingerhut, B. P., Sailer, C. F., Ammer, J., Riedle, E. \& de Vivie-Riedle, R. Buildup and decay of the optical absorption in the ultrafast photo-generation and reaction of benzhydryl cations in solution. J. Phys. Chem. A 116, 11064-11074 (2012).

35. Sailer, C. F. et al. A comprehensive microscopic picture of the benzhydryl radical and cation photogeneration and interconversion through electron transfer. ChemPhysChem 14, 1423-1437 (2013).

36. Ortega, J., Rafols, C., Bosch, E. \& Roses, M. Solute-solvent and solvent-solvent interactions in binary solvent mixtures. Part 3. The $\mathrm{E}_{\mathrm{T}}(30)$ polarity of binary mixtures of hydroxylic solvents. J. Chem. Soc. Perkins Trans. 2, 1497 (1996).

37. Jorgensen, W. L., Maxwell, D. S. \& Tirado-Rives, J. Development and testing of the OPLS all-atom force field on conformational energetics and properties of organic liquids. J. Am. Chem. Soc. 118, 11225-11236 (1996).

38. Sherwood, P. et al. QUASI: a general purpose implementation of the QM/MM approach and its application to problems in catalysis. J. Mol. Struct. THEOCHEM 632, 1-28 (2003).

39. Metz, S., Kästner, J., Sokol, A. A., Keal, T. W. \& Sherwood, P. ChemShell-a modular software package for QM/MM simulations: ChemShell. WIREs Comput. Mol. Sci. 4, 101-110 (2014).

40. Zoete, V., Cuendet, M. A., Grosdidier, A. \& Michielin, O. SwissParam: a fast force field generation tool for small organic molecules. J. Comput. Chem. 32, 2359-2368 (2011).

41. Vanquelef, E. et al. R.E.D. Server: a web service for deriving RESP and ESP charges and building force field libraries for new molecules and molecular fragments. Nucleic Acids Res. 39, W511-W517 (2011).

42. Dupradeau, F.-Y. et al. The R.E.D. tools: advances in RESP and ESP charge derivation and force field library building. Phys. Chem. Chem. Phys. 12, 7821 (2010).

43. Frisch, M. J. et al. Gaussian 09 Revision D.01 (Gaussian, Inc., 2009).

44. Phillips, J. C. et al. Scalable molecular dynamics with NAMD. J. Comput. Chem. 26, 1781-1802 (2005).
45. Becke, A. D. Density-functional thermochemistry. III. The role of exact exchange. J. Chem. Phys. 98, 5648-5652 (1993).

46. Lee, C., Yang, W. \& Parr, R. G. Development of the Colle-Salvetti correlationenergy formula into a functional of the electron density. Phys. Rev. B 37, 785-789 (1988).

47. Grimme, S., Ehrlich, S. \& Goerigk, L. Effect of the damping function in dispersion corrected density functional theory. J. Comput. Chem. 32, 1456-1465 (2011).

48. Eichkorn, K., Weigend, F., Treutler, O. \& Ahlrichs, R. Auxiliary basis sets for main row atoms and transition metals and their use to approximate Coulomb potentials. Theor. Chem. Acc. 97, 119-124 (1997).

49. Ahlrichs, R., Bär, M., Häser, M., Horn, H. \& Kölmel, C. Electronic structure calculations on workstation computers: the program system turbomole. Chem. Phys. Lett. 162, 165-169 (1989).

50. Todorov, I. T. \& Smith, W. DL_POLY_3: the CCP5 national UK code for molecular-dynamics simulations. Phil. Trans. R. Soc. Lond. A 362, 1835-1852 (2004).

51. Senn, H. M. \& Thiel, W. QM/MM methods for biomolecular systems. Angew. Chem. Int. Ed. 48, 1198-1229 (2009).

52. Weigend, F., Häser, M., Patzelt, H. \& Ahlrichs, R. RI-MP2: optimized auxiliary basis sets and demonstration of efficiency. Chem. Phys. Lett. 294, 143-152 (1998).

53. Inamoto, K., Saito, T., Katsuno, M., Sakamoto, T. \& Hiroya, K. Palladiumcatalyzed $\mathrm{C}-\mathrm{H}$ activation/intramolecular amination reaction: a new route to 3-aryl/alkylindazoles. Org. Lett. 9, 2931-2934 (2007).

54. Coudreau, S., Kaplan, D. \& Tournois, P. Ultraviolet acousto-optic programmable dispersive filter laser pulse shaping in KDP. Opt. Lett. 31, 1899-1901 (2006).

55. Linden, S., Giessen, H. \& Kuhl, J. XFROG - a new method for amplitude and phase characterization of weak ultrashort pulses. Phys. Status Solidi B 206, 119-124 (1998).

56. Nuernberger, P. et al. Generation of shaped ultraviolet pulses at the third harmonic of titanium-sapphire femtosecond laser radiation. Appl. Phys. B 88, 519-526 (2007).

57. Megerle, U., Pugliesi, I., Schriever, C., Sailer, C. F. \& Riedle, E. Sub-50 fs broadband absorption spectroscopy with tunable excitation: putting the analysis of ultrafast molecular dynamics on solid ground. Appl. Phys. B $\mathbf{9 6}$, 215-231 (2009).

\section{Acknowledgements}

We thank Tobias Brixner for supporting the experiments. We further acknowledge the German Research Foundation for support within the Cluster of Excellence RESOLV EXC1069, the collaborative research center SFB1093 (E.S.-G.), and the Emmy-Noether program (P.N.). E.S.-G. acknowledges the support of the Boehringer Ingelheim Foundation (Plus-3 Program)

\section{Author contributions}

W.S., E.S.-G. and P.N. conceived the research; J.K., S.S. and P.N. performed and analysed experiments; P.S. and E.S-G. performed and analysed simulations; P.C. performed synthesis; all authors contributed to the manuscript preparation.

\section{Additional information}

Supplementary Information accompanies this paper at http://www.nature.com/ naturecommunications

Competing financial interests: The authors declare no competing financial interests.

Reprints and permission information is available online at http://npg.nature.com/ reprintsandpermissions/

How to cite this article: Knorr, J. et al. Competitive solvent-molecule interaction govern primary processes of diphenylcarbene in solvent mixtures. Nat. Commun. 7, 12968 doi: 10.1038/ncomms12968 (2016).

This work is licensed under a Creative Commons Attribution 4.0 International License. The images or other third party material in this article are included in the article's Creative Commons license, unless indicated otherwise in the credit line; if the material is not included under the Creative Commons license, users will need to obtain permission from the license holder to reproduce the material. To view a copy of this license, visit http://creativecommons.org/licenses/by/4.0/

(C) The Author(s) 2016 